\title{
A analysis of comparative study on intramedullary locking nail and plating for periarticular fractures of distal tibia
}

\author{
Mathun Ram ${ }^{1, *}$, Bharath ${ }^{2}$, Sundharaja ${ }^{3}$ \\ ${ }^{1}$ Post Graduate, ${ }^{2,3}$ Assistant Professor, Dept. of Orthopaedic, Sri Ramachandra Medical College, SRM University, Chennai,
} Tamil Nadu, India

*Corresponding Author: Mathun Ram

Email: nathanorthocare@gmail.com

\begin{abstract}
Aim and Objective: Our aim is to find out the best line of treatment both radiologically and clinically for the extra articular fractures of the distal tibia treated with imil nailing and plating.

Materials and Methods: From 2016 may till 2018 January all patients with extra articular fractures were taken for the study. All are closed fractures.17 patients under went imil nailing and 15 underwent distal tibia plating patients with diabetes, smokers, alcoholics were included in relation to wound healing. Results were analysed as per the scoring the two sets of patients were analysed for infection, skin necrosis, non-union, malunion, ankle rom, knee pain, shortening etc.

Results: in plating no infection, skin necrosis in one patient, non-union noticed in 3 patients and finally in 9months all united except one malunion is more in plating, ankle rom, stiffness is noticed knee pain noticed in few cases of imil nailing cases, shortening noticed in one case in plating results are graded as excellent, good, fair, and poor. In nailing-no infection, no skin necrosis, nononunion, no angulation, ankle rom full, knee pain in 1case,and no shortening results were graded as excellent, good, fair and poor both groups were compared.

Discussion: Imil cases bone united in $8 \mathrm{w}$, early weight bearing, no infection, skin necrosis, no angulation, no shortening noticed. In plating shortening, delayed union, angulation, ankle stiffness which is not seen in imil smoking diabetes, and alcoholics landed up in skin necrosis and plate exposures.

Conclusion: In our study we found imil nailing is the best and less complication and early reture to work it gives early weight bearing, no shortening, no delayed /non-union, no infection, early union and no malunion rom at ankle is very good rarely knee pain. Hence we conclude imil gives the best result.

In our study the fractures were operated by different surgeons. We fell a bigger randomised trial involving more number of cases will show how the imil is superior to the plates.
\end{abstract}

Keywords: Tibial fracture, Imil nail, Plating tibia, Distal tibial.

\section{Introduction}

Due to the Anatomy $(40,41)$ of distal tibia, extra articular fractures are different. Distal fracture of tibia fracture occurs as a result of axial and rotational force occurring $10 \%$ of fracture. Bone is below the skin with our muscle will poor varcularity compare to other areas. Here the bone is subcutaneous with depleted muscular cover. The varcularity of this region is also scanty compared to the other regions of long bones 44 . A vital aspect that may contribute to infection, wound dehiscence, delayed union or worse non-union of the fracture segments. This fracture above ankle font is complex than a deaphyseal or middle injuries. It is completely different from the pilon fractures 43,45 in terms of the mechanism of injury, degree of displacement of the fragments, its management and also the prognosis, thus resulting in problematic functional and radiological outcomes more often. There may also be surgical challenges due to its anatomy. Reduction becomes difficult because of a wide metaphysis, suboptimal skin or communition of the fracture. Itself which may be further complicated by the presence of a broken fibula at the same level rendering it mechanically unstable.
Materials and Methods

Type of Study: Prospective.

Study Period: June 2014 to January 2017.

Study Setting: Sri Ramachandra Medical College and Research Institute, Porur, Chennai.

Subjects: Patients who have been diagnosed to have a closed extra-articular distal third tibia fracture.

Inclusion Criteria: Closed extra-articular distal third tibia fractures (4 to $11 \mathrm{~cm}$ from the tibial plafond).

Exclusion Criteria: Compound fractures, intraarticular Fractures, paediatric fractures and pathological fractures.

Sample Size: 30 .

Data Collection: Prospective patient data will be collected during clinical review as mentioned and documented.

\section{Surgical Technique}

Plating: Minimally Invasive Percutaneous Plate Osteosynthesis (MIPPO)

\section{Preparation}

Supine Position for MIPPO: The patient was placed supine on a radiolucent operating table in neutral position and the foot was brought to the end of the table. Padding under the leg helped to elevate it during lateral fluoroscopy and aided in reduction of the 
fracture segments. The fractured leg was prepared circumferentially from the toes to mid-thigh region and draped free from the rest of the torso. An image intensifier was placed on the opposite side, and moved whenever required for AP and lateral views. Tourniquet was not required.

\section{Approach}

Skin Incision: Slightly curved skin incision was made on the medial aspect of the distal tibia. Its length ranged from 5-6 cm, depending on the type of the planned plate. The incision stopped distally at the tip of the medial malleolus. Separate stab incisions were placed for fixing proximal screws in the diaphysis.

Surgical Dissection: The incision was continued down to the subcutaneous fat, preserving the greater saphenous vein and saphenous nerve. Retractions were done with a blunt retractor placed anteriorly. The dissection was further advanced up to the periosteum and completely preserved. The epiperiosteal anatomic space helped in tunnelling towards the diaphysis and was achieved with the blunt tip of the plate.

\section{Techniques}

Implant Choice: A $3.5 \mathrm{~mm}$ or occasionally even a 4.5 $\mathrm{mm}$ standard or locked plate (LC-DCP or LCP) was used. For distal fractures and in cases of osteoporosis, angular stable screws (locking head screws) were used since they were more stable distally. With MIPPO plate constructs as long an implant as possible was chosen for the widest load distribution at the fracture site.

Plate Contouring: A non-contoured plate was shaped prior to sterilization, using a saw-bone model as a template. Its length was determined preoperatively from $\mathrm{x}$-rays. The plate was twisted to fit the distal tibia snugly. The medial tibia distally lied closer to the sagittal plane as illustrated, while the shaft rotated externally above the metaphysis.

Plate Insertion: After proximal tunnelling with a blunt instrument, the preformed plate was seated. Depending on the fracture location, the plate was FIXED on the antero-medial aspect of the tibia.

Plate Positioning: A small incision of about $2-3 \mathrm{~cm}$ was made above the fracture zone proximally to assist in positioning the pre-formed plate precisely. The plate and proximal screw should be centered on the tibia, especially if the locking screws were to be used. The distal end of the plate was kept in line with the tibial plafond. The proximal end accomodated in three screw holes proximal to the fracture zone.

Preliminary Plate Stabilization: Once optimal plate position was attained, a single conventional "positioning" screw was passed through the plate to bring the plate flush to the bone. Assuming the plate is accurately contoured and placed; fixing the distal tibial segment to the plate completed the fracture reduction perfectly. A second conventional "reduction" screw was generally used to draw the plate and bone close to each other to achieve the final reduction distally. A check shot was made with image intensification. Later, locking head screws (LHS) were engaged into the reduced distal fragment.

\section{Finish Plate Fixation of the Tibia}

As soon as preliminary fixation and reduction were complete to our satisfaction, additional screws were incorporated for better stability. Stab incisions closer to the fracture site was needed to fix these screws. Their number and position depended on its fracture pattern. The ultimate aim was to have "balanced fixation" with equivalent strength in both proximal and distal fragments.

Fixation of the Fibula: Majority of A3-type fractures have concomitant fibula fractures that need fixation. Complex fractures of the fibula were better addressed after stabilizing the tibial bone.

\section{Nailing: Intramedullary Interlocking Nailing (IMIL) \\ Preparation:}

Supine Position for Nailing: On a radiolucent table the patient was allowed to lie supine. The injured leg was positioned with the knee flexed at least $90^{\circ}$. The thigh was supported on a padded rest so as to enable ease of movement and manipulation of the fracture segments. Alternatively, an external fixator or distractor was used to hold the fracture reduced while the foot rested flat on the table. The uninjured leg was extended and draped using standard protocols.

Skin Incision: Longitudinal skin incision from the lower pole patella to the tibial tuberosity was made. The incision was centered over the patellar ligament.

Relationship to the Patellar Ligament: Usually the patellar ligament was either split in the middle, or retracted after dividing the fascia to the medial or lateral side. The entry point was targeted over the tibial medullary canal.

Mobilizing the Fat Pad: Anterior edge of the tibial plateau was identified and the retropatellar fat pad was released from the tibia and moved posteriorly without opening the knee joint.

Determination of the Entry Point: Depending on the nail design, the nail entry point varied on a lateral view. We made careful consideration while choosing a 61 particular nail to be used based on its recommendations. A more proximal entry point avoided a large anterior tibial defect due to reaming. The nail was clear from the patella, but did not penetrate the posterior tibial cortex. It was first directed slightly posteriorly and then redirected along the medullary canal.

Entry Point Location: The entry point was on the anterior edge of the tibial plateau, evading the menisci, and centered over the medullary canal on the AP view.

Entry Site Fluoroscopy: The entry site was marked just medial to the lateral tibial spine on the AP fluoroscopic view. 
Opening the Medullary Canal with an Awl: The sharp tip of the awl was pressed into the cortex at the entry site, aiming and advancing posteriorly, turning the awl back and forth. Care was taken not to stray away from the posterior aspect of the anterior cortex, and was aimed down the medullary canal exactly in the midline on the AP view. Gradually the awl was rotated to align it with the center of the canal on the lateral view. The shaft of the awl was finished parallel to the anterior cortex of the tibia. Patellar tendon was guarded with a retractor.

\section{Technique}

Reduction by Manual Traction: The distal femur was supported by a leg rest. Manual traction to the foot, restored length, and generally corrected any angulation or rotation. Manual support was maintained as the nail was inserted, typically with back pressure against the nail. For very distal fractures, traction pin in the calcaneus, talus or distal tibial region was required to apply adequate manual traction.

Fibular Fracture Reduction: Fixal distal fibula fracture often helps by indirectly reducing the distal tibial fracture via ligamentotaxis.

Ball-tip Guide Wire: During dissection, as soon as the proximal metaphysis was breached, a ball-tip guide wire was passed down the medullary canal and proceeded deep down into the distal metaphysis. We confirmed accurate reduction of the fracture at the time of passing the guide-wire through it and ended as near as the central aspect of the distal articular tibial segment as possible.

Fluoroscopic Control: The position of the guide wire above the centre of the ankle joint was confirmed with fluoroscopy. We ensured if the angular deformity was corrected in both AP and lateral views in case of distal fractures, before proceeding further.
Determination of Nail Length: As a mandatory we placed the nail as deep as possible while fixing distal tibias. Thus the nail was chosen to be long enough by picking an appropriate length or a system that was able to incorporate a proximal extension. However, all the nails were held at an apt length and did not protrude proximally. The length of the uninjured leg served as a guide for this purpose. The length was restored accurately before measurement in comminute fracture.

Use of a Radiographic Ruler: Nail length was determined intra-operatively using a radiographic ruler. The nail length was measured from the centre of the distal tibia to the planned entry after fracture reduction. Measurement result in short nail and necessary precaution to be taken.

Guide-wire Measurement: First the guide wire was inserted across the reduced fracture to its maximal depth and then a second guide wire of equal length, was placed at the entry portal and the difference in length between the two wires were measured. This difference represented the proper nail length.

\section{Results}

Age group of all the 30 participants ranged from 24 to 77 years with an average age of 29.87 years. Predominantly the participants were men with only $27 \%$ female participants. Pre-existing illness included nine patients with diabetes $(\mathrm{p}=.001)$ and five patients with hypertension $(\mathrm{p}=.330)$. Eleven patients had preexisting habits of smoking and seven patients consumed alcohol occasionally. Thirteen patients had 43-A1 fracture classification eleven patients had 43-A3 and six patients displayed 43-A2 ( $\mathrm{p}=.003)$. All patients in the nailing group presented with right sided fractures whereas patients who underwent plating did not have a side predilection.

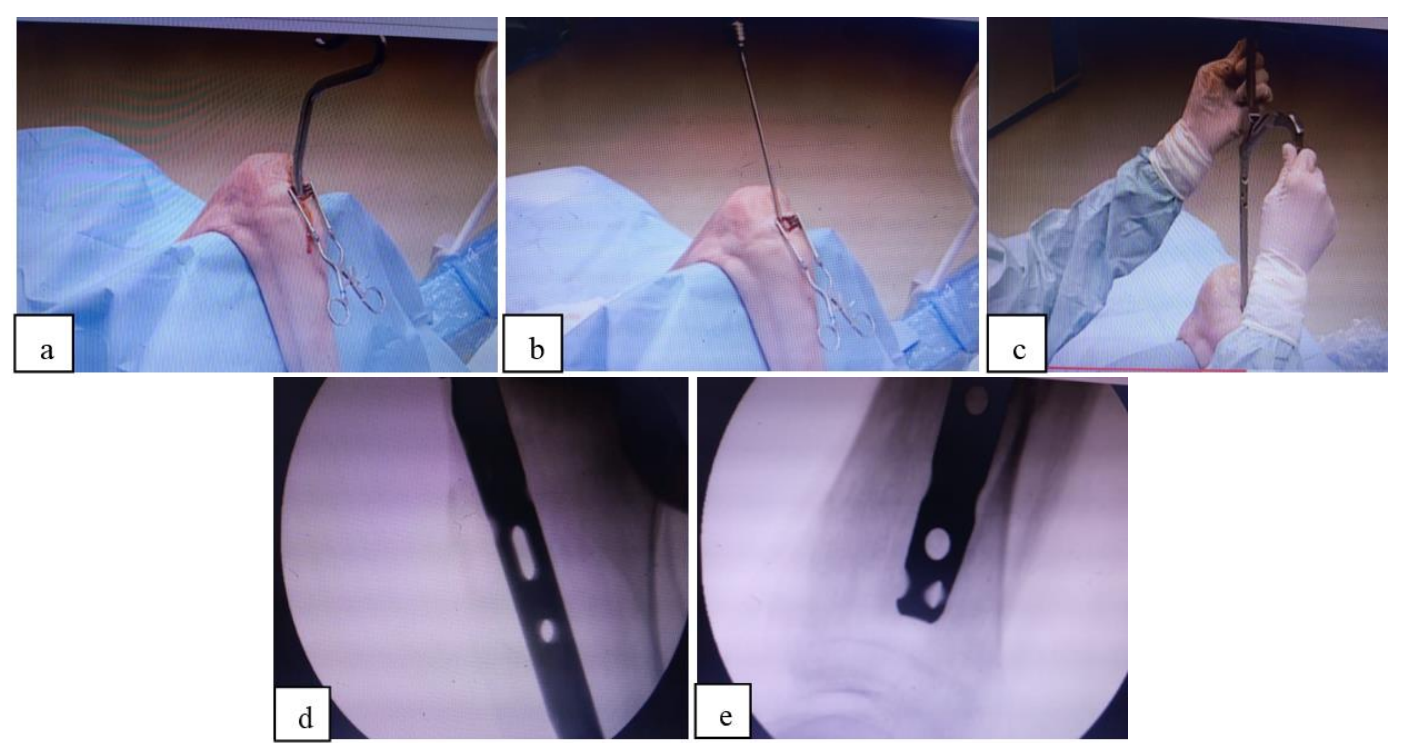

Fig. 1: Interlocking nail (a): Making a Bone hole; (b): Reaming; (c): Nail Insertion; (d): Seeing proximely hole 'C'ARM; (e): Seeing distal hole 

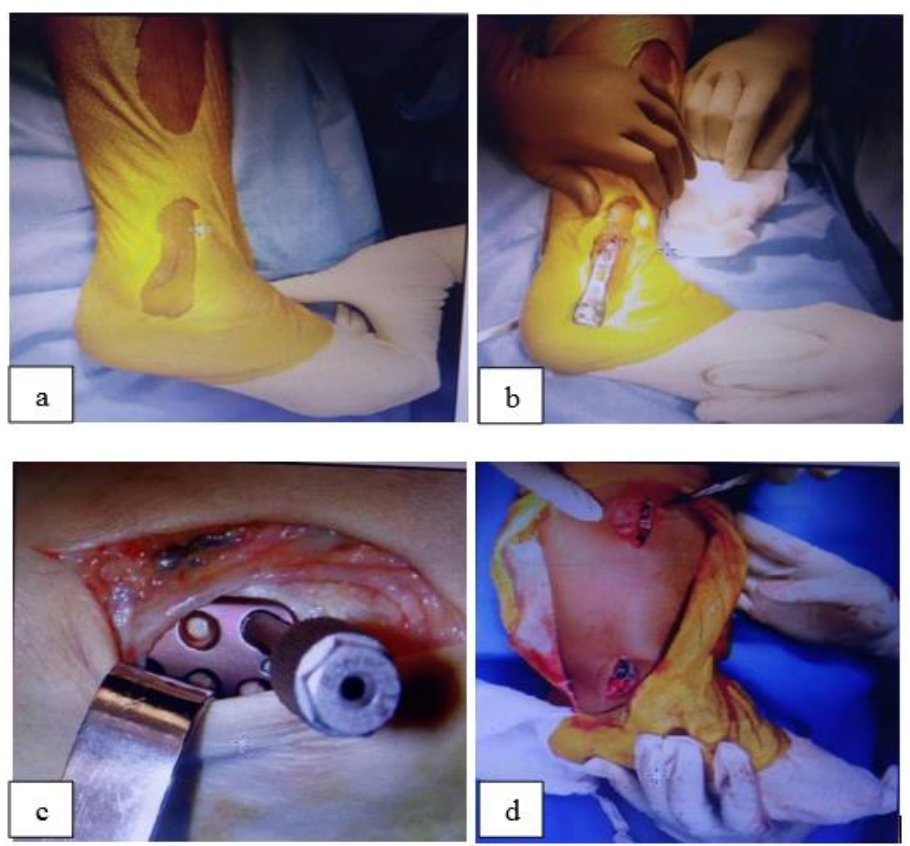

Fig. 2: Minimally invasive locking plate and screws; (a): After drapping and postioning; (b) Plate insertion; (c): Plate insertion with zig; (d): After fixing plate and screws

\section{Discussion}

Muller et al defined the distal tibial metaphysis as the area included within a square, the sides of which are the same length as the widest part of the distal articular surface 39. Earlier Johner and Wruhs classified diaphyseal tibial fractures but this is reversed in metaphyseal fractures84. Fractures in this area were classified by many. The most widely used being the classification proposed by AO39. There is still a controversial debate for the fixation of distal tibial fracture because of the complications involved79. Broadly, tibial intramedullary inter-locking nailing and extramedullary plating are in practice. However, the concept of concomitant fibula fixation may or may not be done. Nailing has been used by many authors to successfully treat this fracture. It comes with limited secondary procedures but at the same time maintain good limb alignment 70 . There may be difficulty in reduction of distal metaphyseal tibial fractures due to the large diameter of the distal part of the tibia relative to the diameter of the intramedullary nail. Also the interface between the cortex of the tibia and the nail cannot be used to assist in fracture reduction. Hence to avoid this difficulty, adjunctive blocking screws may be used to obtain reduction and alignment 75 .

\section{Conclusion}

Intramedullary inter-locking nails and plates deserve a prominent place in treating extra-articular distal third tibia fractures. Results of intramedullary inter-locking nailing showed majority of excellent scores in ten out of fifteen patients. This was followed by two patients with good and three patients with fair grading respectively. Plating resulted in 3 excellent end grades, eight good, three fair and one poor consequence. The union time in intramedullary nailing was short when compared to plating. Placement of guide wire in the center of the bone observed in anteroposterior and lateral views after reduction and also avoiding eccentric reaming played a vital role in avoiding malalignment of the fracture segments while nailing. Generally nailing procedure can be performed earlier that plating due to the presence of swelling.

\section{Conflict of Interest: None}

\section{References}

1. Rockwood CA, Green DP, Bucholz RW, MacQueen MM. Rockwood and Green's fractures in adults. 7thed. Robert W. Bucholz, Charles M. Court - Brown, James D. Heckman, Paul Tornetta III; assoc. ed.: Margaret M. McQueen and William M. Ricci. Philadelphia: Wolters Kluwer/ Lippincott Williams \& Wilkins; 2010.

2. Abitzke R, Weiland A.J, Neuerburg K P S, Otto F, Dall DM, Miles A. Manual of cable osteosyntheses. New York: Springer- Verlag, 2000.

3. Lambotte $\mathrm{A}$. Technique et indication des prothèsesdans le traitement des fractures. Presse Med. 1909;17:321-323.

4. Lane WA. Some remarks on the treatment of fractures. $\mathrm{Br}$ Med J. 1895;1(1790):861-863.

5. Sherman WO. Vanadium steel bone plates and screws. Surg Gynecol Obstet. 1912;14:629-634.

6. Townsend K, Gilfillan C. A new type of bone plate and screws. Surg Gynecol Obstet. 1943;77:595-597.

7. Eggers GW. Internal contact splint. J Bone Joint Surg Am. 1948;30A(1):40-52.

8. Freeland AE, Jabaley ME, Hughes JL.History. In: Freeland AE, Jabaley ME, Hughes JL. Stable fixation of the hand and wrist. New York: Springer-Verlag; 1986.

9. Danis R, Robert. Théorieetpratique de l'ostéosynthèse. Paris: Massonetcie; 1949. https://lib.ugent.be/catalog/rug01:000126769 
10. Venable CS. An impacting bone plate to attain closed coaptation. Ann Surg. 1951;133(6):808-813.

11. Bagby GW, Janes JM. The effect of compression on the rate of fracture healing using a special plate. Am J Surg. 1958;95(5):761-771.

12. AO foundation. Surrey, UK; Newman K.1958 AO Founders https://www.aofoundation.org/.../first50years_historyofth eao_kev innewman.ppt

13. Aksekili MA, Celik I, Arslan AK, Kalkan T, Uğurlu M. The results of minimally invasive percutaneous plate osteosynthesis (MIPPO) in distal and diaphyseal tibial fractures. Acta Orthop Traumatol Turc. 2012;46(3):161167.

How to cite this article: Ram M, Bharath, Sundharaja. A analysis of comparative study on intramedullary locking nail and plating for periarticular fractures of distal tibia. Indian J Orthop Surg. 2018;4(4):375-379. 